\title{
Nonlinear evolution of the centrifugal instability using a semi-linear model
}

\author{
Eunok $\operatorname{Yim}^{1} \dagger$, P. Billant ${ }^{2}$ and F. Gallaire ${ }^{1}$ \\ ${ }^{1}$ LFMI, École Polytechnique Fédérale de Lausanne, 1015 Lausanne,Switzerland \\ ${ }^{2}$ LadHyX, CNRS, École Polytechnique, F-91128 Palaiseau CEDEX, France
}

(Received xx; revised xx; accepted xx)

\begin{abstract}
We study the nonlinear evolution of the centrifugal instability developing on a columnar anticyclone with a Gaussian angular velocity using a semi-linear approach. The model consists in two coupled equations: one for the linear evolution of the most unstable perturbation on the axially averaged mean flow and another for the evolution of the mean flow under the effect of the axially averaged Reynolds stresses due to the perturbation. Such model is similar to the self-consistent model of Mantič-Lugo et al. (2014) except that the time averaging is replaced by a spatial averaging. The non-linear evolutions of the mean flow and the perturbations predicted by this semi-linear model are in very good agreement with DNS for the Rossby number $R o=-4$ and both values of the Reynolds numbers investigated: $R e=800$ and 2000 (based on the initial maximum angular velocity and radius of the vortex). An improved model taking into account the second harmonic perturbations is also considered. The results show that the angular momentum of the mean flow is homogenized towards a centrifugally stable profile via the action of the Reynolds stresses of the fluctuations. The final velocity profile predicted by Kloosterziel et al. (2007) in the inviscid limit is extended to finite high Reynolds numbers. It is in good agreement with the numerical simulations.
\end{abstract}

Key words: Centrifugal instability, vortex, semi-linear model, stability analysis

\section{Introduction}

Centrifugal instability or inertial instability, is the most common instability developing on vortices in rotating medium. It is a local instability occurring when the balance between the centrifugal force and the pressure gradient is disrupted, i.e. when the square of the absolute angular momentum of the fluid decreases with radius $r$ in inviscid fluids (Rayleigh 1917, Synge 1933, Kloosterziel \& van Heijst 1991). While this condition applies to axisymmetric disturbances, a generalized criterion for non-axisymmetric perturbations has been derived by Billant \& Gallaire (2005).

Linear stability analysis of a columnar vortex with Gaussian angular velocity in inviscid fluids shows that the growth rate is maximum at infinite wavenumber (Smyth \& McWilliams 1998). However, as soon as viscous effects are taken into account, short wavelength are damped and the fastest growing mode has a finite wavenumber (Lazar et al.|2013; Yim et al. 2016).

Kloosterziel et al. (2007) and Carnevale et al. (2011) have analysed the nonlinear

$\dagger$ Email address for correspondence: eunok.yim@epfl.ch 
evolution of the centrifugal instability in a rotating medium at high Reynolds number. They have shown that the vortex saturates to a centrifugally stable state where the Rayleigh instability condition is no longer satisfied, i.e. the square of the axial average of the absolute angular momentum does not decrease with radius. Hence, the instability redistributes the regions of positive and negative absolute angular momentum under the constraint of absolute angular momentum conservation in the inviscid limit.

The saturation of an instability towards a periodic limit cycle for which the mean flow is stable has been recently described by means of a self-consistent approach (Mantič-Lugo et al. 2014). In this approach, the flow is decomposed into time-averaged mean flow and unsteady perturbations. Then, the nonlinear saturation can be described by computing the mean flow distortion due to the Reynolds stresses of the perturbation and the linear growth of the perturbation on the mean flow. Here, we develop a similar approach for the centrifugal instability using a spatial average instead of a time average since the instability is spatially periodic but not periodic in time.

\section{Governing equations}

We consider a vortex with angular velocity (figure 1 1 )

$$
\Omega=\Omega_{0} \exp \left(-r^{2} / R^{2}\right),
$$

where $\Omega_{0}$ is the maximum angular velocity and $R$ the radius of the vortex. In the following, the length and time are non-dimensionalised with $R$ and $1 / \Omega_{0}$, respectively. The governing equations for the velocity field $\mathbf{u}=[u, v, w]$ in cylindrical coordinates $(r, \theta, z)$ read

$$
\begin{aligned}
\frac{\partial \mathbf{u}}{\partial t}+\mathbf{u} \cdot \nabla \mathbf{u}+2 R o^{-1} \mathbf{e}_{z} \times \mathbf{u} & =-\nabla p+R e^{-1} \nabla^{2} \mathbf{u}, \\
\nabla \cdot \mathbf{u} & =0,
\end{aligned}
$$

where the Reynolds and Rossby numbers are defined as $R e=\Omega_{0} R^{2} / \nu$ and $R o=2 \Omega_{0} / f$, respectively with $\nu$ the kinematic viscosity and $f$ the Coriolis parameter.

\subsection{Linear stability}

The linear stability of the base flow 2.1 has been first studied by linearizing the equations 2.2 -2.3 and assuming axisymmetric infinitesimal perturbations with axial wavenumber $k$. In the inviscid limit, the necessary and sufficient condition for centrifugal instability reads (Rayleigh 1917; Synge 1933; Kloosterziel \& van Heijst 1991),

$$
\phi=2\left(\frac{v}{r}+\frac{1}{R o}\right)\left(\xi+\frac{2}{R o}\right)<0,
$$

where $\xi=1 / r \partial(r v) / \partial r$ is the axial vorticity. For the base flow 2.1), 2.4 is satisfied when $R o<-1$ and $R o>\exp (2)$. Figure $1 \mathrm{~b}$ shows the linear growth rate $\sigma$ as a function of $k$ for $R o=-4$ for two different Reynolds numbers, $R e=800$ and $R e=2000$. The growth rate is maximum at $k_{m}=5.6$ for $R e=800$ and $k_{m}=8.6$ for $R e=2000$. Figure 1. shows the most unstable eigenmode for $R e=800, R o=-4$. It is mostly localized in the region where the Rayleigh discriminant is negative (shaded area).

\section{Semi-linear formulation}

We decompose the flow as

$$
\mathbf{u}(r, z, t)=\overline{\mathbf{u}}(r, t)+\hat{\mathbf{u}}(r, z, t)
$$



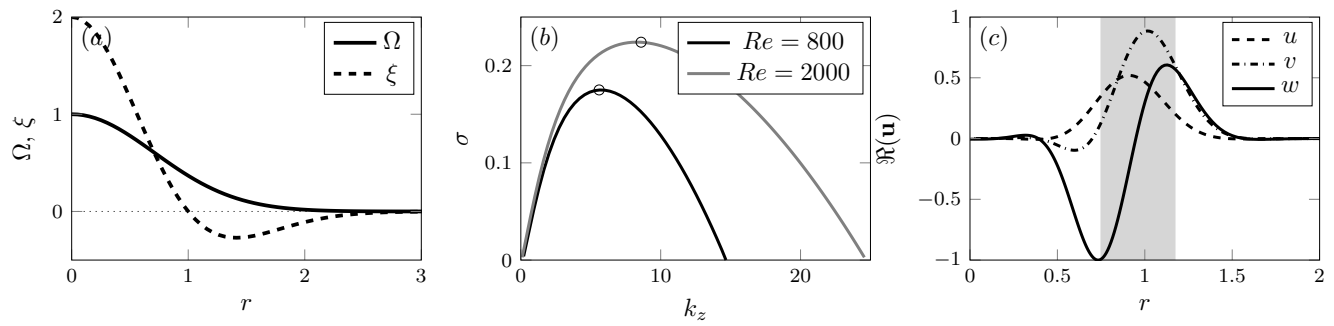

Figure 1. (a) Angular velocity $(\Omega)$ and axial vorticity $(\xi)$ of the base flow. (b) Linear growth rate as a function of the vertical wavelength $k$ for $R e=800$ and $R e=2000$ for $R o=-4$. (c) Real part of the most unstable eigenmode for $R e=800$. The shaded area indicates the region where $\phi<0$.

where $\overline{\mathbf{u}}=z_{\max }^{-1} \int_{0}^{z_{\max }} \mathbf{u d} z$ is the axially averaged mean flow and $\hat{\mathbf{u}}$ the perturbation which is not assumed to be small as in the linear stability analysis. Averaging the equation 2.2 in $z$ leads to

$$
\frac{\partial \overline{\mathbf{u}}}{\partial t}+\overline{\mathbf{u}} \cdot \nabla \overline{\mathbf{u}}+2 R o^{-1} \mathbf{e}_{z} \times \overline{\mathbf{u}}+\nabla \bar{p}-R e^{-1} \nabla^{2} \overline{\mathbf{u}}=-\overline{\hat{\mathbf{u}} \cdot \nabla \hat{\mathbf{u}}} .
$$

Substracting $\sqrt{3.2}$ from $(2.2$ yields the equation for the perturbation $\hat{\mathbf{u}}$,

$$
\frac{\partial \hat{\mathbf{u}}}{\partial t}+\overline{\mathbf{u}} \cdot \nabla \hat{\mathbf{u}}+\hat{\mathbf{u}} \cdot \nabla \overline{\mathbf{u}}+2 R o^{-1} \mathbf{e}_{z} \times \hat{\mathbf{u}}+\nabla \hat{p}-R e^{-1} \nabla^{2} \hat{\mathbf{u}}=-\hat{\mathbf{u}} \cdot \nabla \hat{\mathbf{u}}+\overline{\hat{\mathbf{u}} \cdot \nabla \hat{\mathbf{u}}}
$$

\subsection{Single harmonic}

We introduce now the normal mode form of the perturbation $\hat{\mathbf{u}}(r, z, t) \simeq$ $\mathbf{u}_{1}(r, t) \exp \left(\mathrm{i} k_{m} z\right)+$ c.c. where c.c indicates the complex conjugate and $k_{m}$ is the most amplified wavenumber obtained from the linear stability analysis. At $t=0$, the perturbation is set as $\mathbf{u}_{1}(r, 0)=A_{0} \mathbf{u}_{m}$ where $\mathbf{u}_{m}$ is the dominant eigenmode and $A_{0}$ the initial amplitude of the perturbation. Neglecting the higher harmonics, the governing equations (3.2)- 3.3) reduce to

$$
\begin{aligned}
\frac{\partial \overline{\mathbf{u}}}{\partial t}+\overline{\mathbf{u}} \cdot \nabla \overline{\mathbf{u}}+2 R o^{-1} \mathbf{e}_{z} \times \overline{\mathbf{u}}+\nabla \bar{p}-R e^{-1} \nabla^{2} \overline{\mathbf{u}} & =-\zeta\left(\mathbf{u}_{1}\right), \\
\frac{\partial \mathbf{u}_{1}}{\partial t}+\mathcal{L}(\overline{\mathbf{u}}) \mathbf{u}_{1} & =0
\end{aligned}
$$

where $\mathcal{L}(\overline{\mathbf{u}})$ is a linear operator defined as $\mathcal{L}(\overline{\mathbf{u}}) \hat{\mathbf{u}} \equiv \overline{\mathbf{u}} \cdot \nabla_{k} \hat{\mathbf{u}}+\hat{\mathbf{u}} \cdot \nabla \overline{\mathbf{u}}+2 R o^{-1} \mathbf{e}_{z} \times \hat{\mathbf{u}}+$ $\nabla_{k} \hat{p}-R e^{-1} \nabla_{k}^{2} \hat{\mathbf{u}}$ and $\zeta\left(\mathbf{u}_{1}\right)=\mathbf{u}_{1} \cdot \nabla_{-k} \mathbf{u}_{1}^{*}+\mathbf{u}_{1}^{*} \cdot \nabla_{k} \mathbf{u}_{1}$ is the Reynolds stress. Here, $\nabla_{k}$ and $\nabla_{k}^{2}$ are respectively the gradient and Laplacian in cylindrical coordinates with the vertical derivative replaced by $\mathrm{i} k$. It is worth mentioning that $(3.4)-(3.5)$ are now only a function of time and the radial coordinate $r$. In addition, the divergence free condition reduces to $1 / r \partial r \bar{u} / \partial r=0$ since $\partial \bar{w} / \partial z=0$ due to the axial averaging. This implies $\bar{u}=0$. It can also be shown that $\bar{w}$ remains identically zero for all time if $\bar{w}=0$ at $t=0$, since the Reynolds stress in the $z$ direction is zero. Thus, the mean flow has only a component along the azimutal direction $\overline{\mathbf{u}}=[0, \bar{v}, 0]^{T}$. Hence, (3.4) simplifies to

$$
\frac{\partial \bar{v}}{\partial t}=R e^{-1}\left[\frac{\partial^{2} \bar{v}}{\partial r^{2}}+\frac{1}{r} \frac{\partial \bar{v}}{\partial r}-\frac{\bar{v}}{r^{2}}\right]-\zeta\left(\mathbf{u}_{1}\right)_{\theta},
$$

which is a simple diffusion equation with a source term independent from the Rossby number $R o$.

Therefore, our model consists in the semi-linear 1D equation 3.5 for the evolution 
of the perturbation over the mean flow coupled to the equation (3.6) for the evolution of the mean flow under the effects of the Reynolds stresses of the perturbation and viscous diffusion. Such semi-linear model is similar to the self-consistent model proposed by Mantič-Lugo et al. (2014). The main difference is that the Reynolds decomposition (Reynolds \& Hussain 1972) to separate the flow into a mean flow and a fluctuation is here based on spatial axial average since the perturbation is harmonic along the axis while the self-consistent model relies upon a time average because the perturbation is harmonic in time for the flows they have considered. Another difference is that the perturbation equations are here simply integrated in time while, in the self-consistent model, an eigenvalue problem has to be solved after each variation of the mean flow.

\subsection{Two harmonics}

One can easily include higher harmonics of the fundamental mode following the same approach. For instance, taking into account the second harmonic in the velocity perturbation: $\hat{\mathbf{u}}=\mathbf{u}_{1}(r) \exp \left(\mathrm{i} k_{m} z\right)+\mathbf{u}_{2}(r) \exp \left(\mathrm{i} 2 k_{m} z\right)+$ c.c., the perturbation equations become

$$
\begin{array}{r}
\frac{\partial \mathbf{u}_{1}}{\partial t}+\mathcal{L}(\overline{\mathbf{u}}) \mathbf{u}_{1}=-\left(\mathbf{u}_{2} \cdot \nabla \mathbf{u}_{1}^{*}+\mathbf{u}_{1}^{*} \cdot \nabla \mathbf{u}_{2}\right) \\
\frac{\partial \mathbf{u}_{2}}{\partial t}+\mathcal{L}(\overline{\mathbf{u}}) \mathbf{u}_{2}=-\left(\mathbf{u}_{1} \cdot \nabla \mathbf{u}_{1}\right)
\end{array}
$$

The mean flow (3.6) is then forced by the Reynolds stress of both harmonics

$$
\frac{\partial \bar{v}}{\partial t}=R e^{-1}\left[\frac{\partial^{2} \bar{v}}{\partial r^{2}}+\frac{1}{r} \frac{\partial \bar{v}}{\partial r}-\frac{\bar{v}}{r^{2}}\right]-\zeta\left(\mathbf{u}_{1}\right)_{\theta}-\zeta\left(\mathbf{u}_{2}\right)_{\theta} .
$$

Higher harmonics $3 k_{m}, 4 k_{m}, \cdots$ can be taken into account similarly. Since the perturbations equation are integrated in time, there is no particular complexity arising when several harmonics are considered. This is in contrast with the self-consistent model (Mantič-Lugo et al. 2014) where the eigenvalue problems become increasingly complicated when more than one harmonic is considered (Meliga 2017).

\subsection{Numerical method}

All numerical simulations have been performed with the FreeFEM++ software (Hecht 2012). The velocity and pressure are discretised with Taylor-Hood P2 and P1 elements, respectively. The time and the convection operators are discretised using characteristicGarleken method for the direct numerical simulations (DNS) while the first order backward Euler formula is used for the semi-linear models. The total numbers of degree of freedom is 278817 for the DNS and 4687 for the semi-linear models, i.e. about 60 times less than for DNS. Both DNS and semi-linear models are initialized with the perturbation $\hat{\mathbf{u}}=A_{0} \mathbf{u}_{m} \exp \left(\mathrm{i} k_{m} z\right)+c . c$. where $A_{0}=0.001$ is the initial amplitude and $\mathbf{u}_{m}$ is the most unstable linear eigenmode (figure 1 ; ) obtained by means of the restarted Arnoldi method. The eigenmodes have been normalized so that the absolute maximum value of the vertical velocity is unity $\max (|w|)=1$.

The domain size is chosen to be $r=\left[0, r_{\max }\right]$ and $z=\left[0, z_{\max }\right]$ where $r_{\max }=8, z_{\max }=$ $2 \pi / k_{m}$ and $k_{m}$ is the most amplified axial wavenumber from the linear analysis. Periodic boundary conditions are applied on $z=0$ and $z=z_{\max }$. The boundary conditions at $r=0$ are $u=v=0$ since the flow is axisymmetric (Batchelor \& Gill 1962). At $r=r_{\max }$, all perturbations are enforced to vanish. Some DNS and simulations with the semi-linear models have been successfully checked against simulations with an independent pseudospectral code NS3D (Deloncle et al. 2008). 

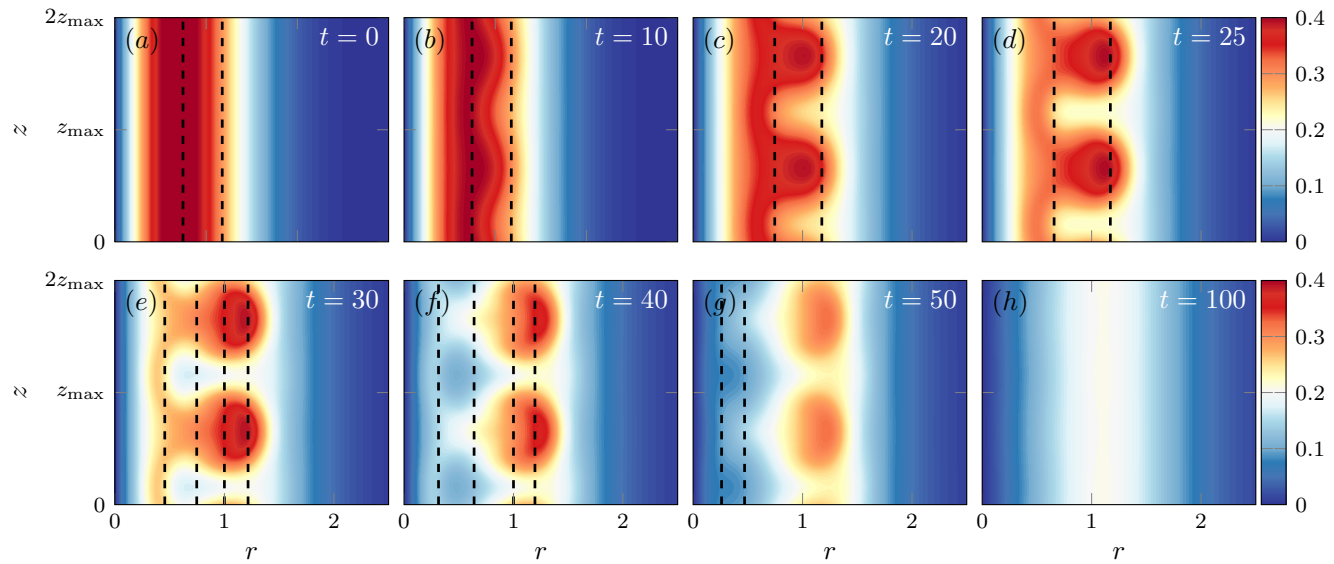

FiguRE 2. Evolution of the azimuthal velocity $v$ in DNS for $k_{m}=5.6, z_{\max }=2 \pi / k_{m}, R o=-4$, $R e=800, A_{0}=0.001$. The dashed lines delimit the regions where $\bar{\phi}<0$ based on the mean azimuthal velocity $\bar{v}$.

\section{Results}

\section{1. $D N S$}

Figure 2 shows snapshots of the azimuthal velocity $v$ in a DNS for $R o=-4$ and $R e=$ 800. Two wavelengths are displayed although the computation is performed over only one wavelength. The vertical lines delimit the regions where the Rayleigh discriminant $\bar{\phi}$ is negative, where $\bar{\phi}$ is based on the axially averaged azimuthal velocity $\bar{v}(t, r)$. At $t=10$, a slight deformation can be seen in the region where $\bar{\phi}<0$. Subsequently, the perturbation grows and rearranges the distribution of azimuthal velocity $(20<t<30)$. For $t>30$, the 'mushrooms' start to fade out. Finally, vertical deformations are no longer visible by $t=100$ so that the vortex then evolves only by viscous diffusion.

The solid lines in figure 3 show the evolution of the corresponding mean azimuthal velocity $\bar{v}$. The mean flow profile first decays by viscous diffusion until $t=10$. A distortion of the mean flow due to the instability can be seen at $t=20$. At $t=30$ and $t=40$, it becomes strong and the profile exhibits two distinct peaks near $r=0.4$ and $r=1$. Then, the peak at $r \sim 0.4$ disappears and the mean azimuthal velocity profile becomes linear for $r<1$ for $t>60$. During this process, the maximum velocity has decreased from $\max (\bar{v}(t=0))=0.47$ to $\max (\bar{v}(t=80))=0.23$ and the corresponding radius has moved from $r=0.7$ to $r=1.2$. The corresponding Rayleigh discriminant is shown in figure $3 \mathrm{~b}$ (solid lines). At $t=0, \bar{\phi}$ is minimum at $r=0.95$ and is negative for $0.75<r<1.18$. For $t=20$, the region where $\bar{\phi}<0$ has enlarged but the minimum of $\bar{\phi}$ has decreased in absolute value. At $t=30$, there exist two regions where $\bar{\phi}<0$ : near $r=0.3$ and $r=0.8$ while $\bar{\phi}>0$ in between. The minimum value decreases and then increases again for larger time. At $t=60, \min (\bar{\phi})$ is no longer negative.

The evolution of the mean azimuthal velocity $\bar{v}$ is qualitatively similar for the higher Reynolds number $R e=2000$ (solid lines in figure 4 a).

\section{2. $S L-1 k_{m}$ semi-linear model}

The lines with symbols in figure 3 a represent the mean azimuthal flow predicted by the semi-linear model with one harmonic, abbreviated as SL- $1 k_{m}$, for $R e=800$. The agreement with the DNS is almost perfect for $t=10,20$ while some discrepancies can be seen at $t=30,40$. It becomes excellent again for $t>60$. Similar agreement and 

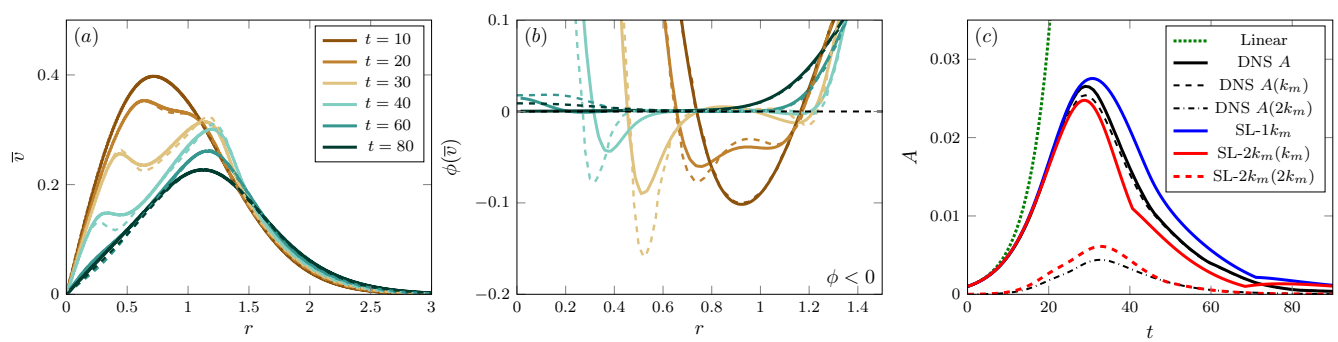

Figure 3. (a) Mean azimuthal velocity $\bar{v}$ from DNS (solid lines) and SL- $1 k_{m}$ model (dashed lines with symbols), (b) corresponding Rayleigh discriminant $\bar{\phi}$ and (c) perturbation amplitudes $A$ as a function of time for $R o=-4$ and $R e=800$.
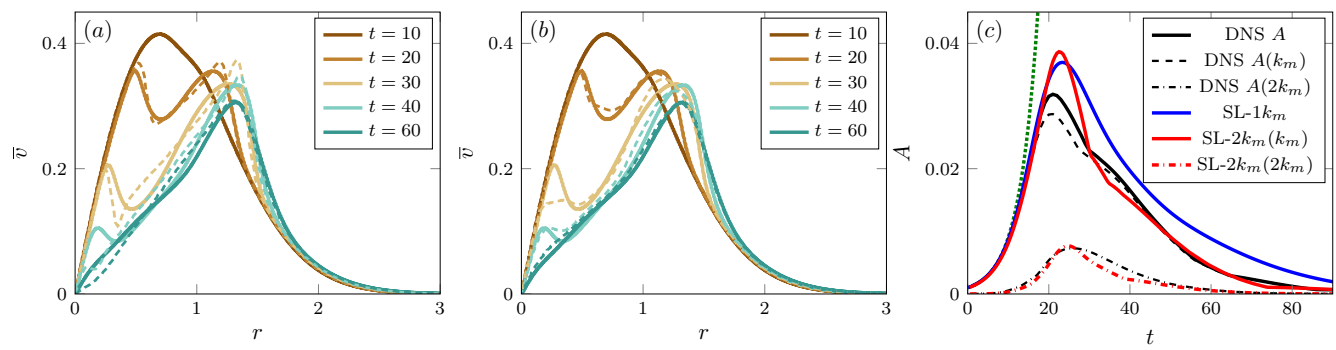

Figure 4. (a) Mean azimuthal velocity from DNS (solid lines) and semi-linear model SL- $1 k_{m}$ (symbols) and (b) for SL- $2 k_{m}$. (c) The perturbation amplitudes $A$ as a function of time for $R o=-4$ and $R e=2000$ for both SL- $1 k_{m}$ and SL- $2 k_{m}$.

discrepancies are also observed for the Rayleigh discriminant $\bar{\phi}$ (figure $3 \mathrm{~b}$ ). Figure 3 . shows the amplitude of the velocity perturbation in the DNS and the semi-linear model. The amplitude first increases exponentially from its initial value $A_{0}=0.001$. The linear prediction (dotted line) agrees with the DNS only for small time $(t<10)$. Then, the growth rate becomes smaller than the linear growth rate. The perturbation grows until $t=30$ and then decreases. The amplitude of the first and second harmonics $A\left(k_{m}\right)$, $A\left(2 k_{m}\right)$ have been decomposed using FFT (broken lines). The amplitude of the second harmonic is less than $20 \%$ of the amplitude of the first harmonic. The amplitude of the perturbation in the semi-linear model SL- $1 k_{m}$ (blue thick line) is in very good agreement with the one in the DNS until $t=30$. Then, it slightly overestimates the amplitude extracted from the DNS.

For the higher Reynolds number $R e=2000$, the evolution of the mean azimuthal flow and amplitudes of the perturbation in the DNS and SL- $1 k_{m}$ model are also globally in good agreement (figure $4 \mathrm{a}, \mathrm{c}$ ). Nevertheless, some deviations can be seen at $t=30,40$, in particular the first peak at small radius is not captured at $t=40$. In addition, the maximum amplitude of the second harmonic reaches $1 / 3$ of the maximum amplitude of the fundamental harmonic (figure 4 c).

\section{3. $S L-2 k_{m}$ semi-linear model}

As seen in figures $3 c$ and $4 k$, the second harmonic is triggered and reaches a nonnegligible amplitude for both $R e=800$ and $R e=2000$. Its effect can be taken into account by means of the semi-linear model $3.7-3.8)$, called SL- $2 k_{m}$. Since the perturbation is initialized by only the leading eigenmode, the initial amplitude of the second harmonic is set to zero. Therefore, even if it is also unstable for $R e=2000$ (figure 1b), its initial evolution is only due to the forcing by the fundamental harmonic. 


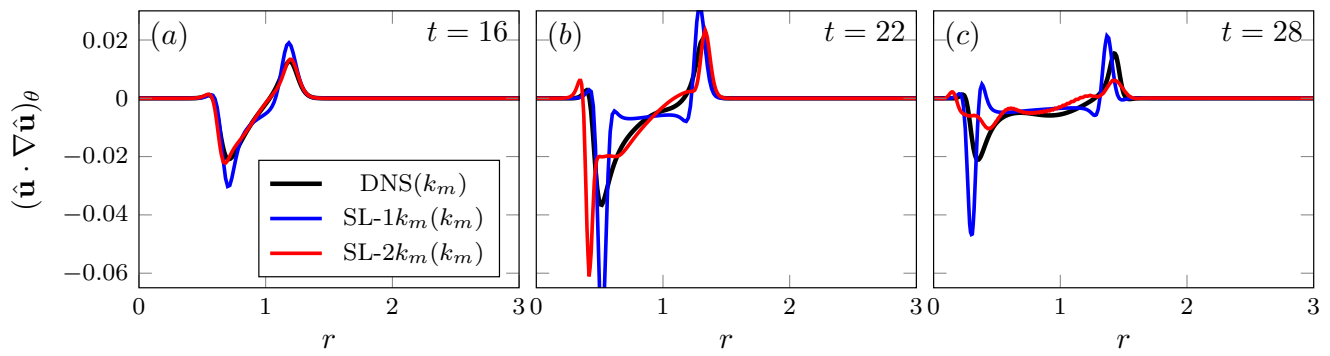

FiguRE 5. Mean Reynolds stresses in the azimuthal direction from DNS and semi-linear models at (a) $t=16$, (b) $t=22$ and (c) $t=28$.

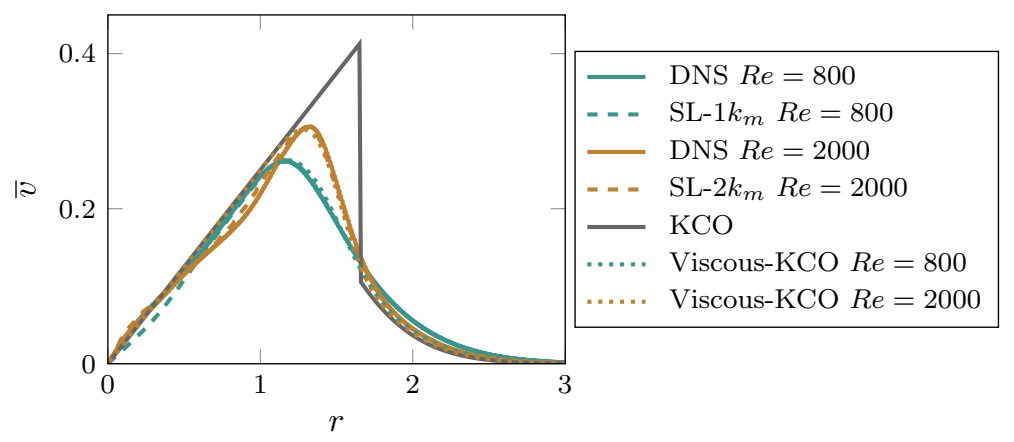

FIgURE 6. Mean azimuthal velocity in the DNS and semi-linear models at $t=60$ for $R o=-4$ for $R e=800$ and $R e=2000$. KCO indicates the velocity distribution predicted by Kloosterziel et al. (2007) in the inviscid limit. Viscous-KCO corresponds to the velocity profile (5.11) which takes into account viscous effects.

For $R e=800$ (figure 3c), the evolution of the amplitude of the first harmonic predicted by the SL- $2 k_{m}$ model is closer to the DNS than for the SL- $1 k_{m}$ model. The amplitude of the second harmonic is also well predicted by the SL- $2 k_{m}$ model.

For $R e=2000$ (figure 4k), the predicted profiles for the mean azimuthal velocity are smoother than for the SL-1 $k_{m}$ model (figure 4 a). However, the first peak near $r \sim 0.3$ at $t=30$ is not captured. To understand this discrepancy, we have plotted the Reynolds stresses in the $\theta$-direction in the DNS and the semi-linear models (figure 5).

At $t=16, \mathrm{SL}-2 k_{m}$ are in excellent agreement with DNS while at $t=22$ and $t=28$, the SL- $2 k_{m}$ model agrees generally better with the DNS except the first minimum at small radius at $t=22$. This explains why the SL- $2 k_{m}$ model does not capture the first peak of the mean azimuthal velocity (figure $4 \mathrm{~b}$ ).

Of course, including higher harmonics should further improve the predictions. However, this would complexify the models while the primary goal of the present approach is the simplicity rather than the accuracy.

\section{Final profiles of azimuthal velocity}

The profiles of mean azimuthal velocity observed at late time $t=60$, once the instability has ceased, have been compared to the theory of Kloosterziel et al. (2007) and Carnevale et al. (2011). As mentioned in the introduction, this theory states that the centrifugal instability homogenizes negative and positive absolute angular momentum $L=r(v+r / R o)$ so as to suppress negative gradients of $L^{2}$ under the constraint of absolute angular momentum conservation. For anticyclones, the final profile of $L$ in the inviscid 
limit is such that $L$ is zero until a radius $r_{c}$ given by $\int_{0}^{r_{c}} r L_{i} \mathrm{dr}=0$, where $L_{i}$ is the initial absolute angular momentum. Beyond this radius, the velocity profile remains identical to the initial one. Therefore, the theoretical angular velocity is

$$
\Omega\left(r<r_{c}\right)=-\frac{1}{R o}, \quad \Omega\left(r \geqslant r_{c}\right)=\exp \left(-r^{2}\right) .
$$

This profile (labelled KCO) is compared to those observed in the DNS and SL models for $R e=800$ and $R e=2000$ in figure 6. It is close to the observed profiles except in the vicinity of the radius $r_{c}$ where the latter are smooth while the theoretical profile is discontinuous due to the inviscid approximation. In order to take into account viscous effects, we have further considered the viscous diffusion of (5.1). For large Reynolds number, the diffusion equation

$$
\frac{\partial \Omega}{\partial t}=R e^{-1}\left[\frac{\partial^{2} \Omega}{\partial r^{2}}+\frac{3}{r} \frac{\partial \Omega}{\partial r}\right]
$$

shows that the angular velocity should decay slowly everywhere except in the vicinity of $r_{c}$ where radial derivatives are expected to be large because of the discontinuity. To describe the local viscous evolution near $r_{c}$, we therefore define a rescaled radial coordinate $\tilde{r}=\sqrt{R e}\left(r-r_{c}\right)$ and we assume that $\Omega$ depends both on $\tilde{r}$ and the unscaled radius $\hat{r}=r$. We also introduce a slow time $\tau=R e^{-1} t$. Hence, 5.2 becomes

$$
\frac{\partial \Omega}{\partial t}+\frac{1}{R e} \frac{\partial \Omega}{\partial \tau}=\frac{\partial^{2} \Omega}{\partial \tilde{r}^{2}}+\frac{1}{\sqrt{R e}}\left(2 \frac{\partial^{2} \Omega}{\partial \tilde{r} \partial \hat{r}}+\frac{3}{\hat{r}} \frac{\partial \Omega}{\partial \tilde{r}}\right)+\frac{1}{R e}\left(\frac{\partial^{2} \Omega}{\partial \hat{r}^{2}}+\frac{3}{\hat{r}} \frac{\partial \Omega}{\partial \hat{r}}\right) .
$$

Then, the solution is sought as an expansion in Reynolds number

$$
\Omega=\Omega_{0}+R e^{-1 / 2} \Omega_{1}+R e^{-1} \Omega_{2}+\cdots .
$$

The zero-th and first order problems are

$$
\frac{\partial \Omega_{0}}{\partial t}=\frac{\partial^{2} \Omega_{0}}{\partial \tilde{r}^{2}}, \quad \text { and } \quad \frac{\partial \Omega_{1}}{\partial t}-\frac{\partial^{2} \Omega_{1}}{\partial \tilde{r}^{2}}=2 \frac{\partial^{2} \Omega_{0}}{\partial \tilde{r} \partial \hat{r}}+\frac{3}{\hat{r}} \frac{\partial \Omega_{0}}{\partial \tilde{r}} .
$$

The solutions are chosen as

$$
\Omega_{0}=A(\hat{r}, \tau) \operatorname{erf}\left(\frac{\tilde{r}}{2 \sqrt{t}}\right)+H(\hat{r}, \tau), \quad \text { and } \quad \Omega_{1}=\left(2 \frac{\partial A}{\partial \hat{r}}+3 \frac{A}{\hat{r}}\right) \sqrt{\frac{t}{\pi}} \exp \left(-\frac{\tilde{r}^{2}}{4 t}\right) .
$$

where $A$ and $H$ are arbitrary functions of $\hat{r}$ and $\tau$. These functions are found by considering the problem at order $R e^{-1}$ :

$$
\frac{\partial \Omega_{2}}{\partial t}-\frac{\partial^{2} \Omega_{2}}{\partial \tilde{r}^{2}}=-\frac{\partial \Omega_{0}}{\partial \tau}+\frac{\partial^{2} \Omega_{0}}{\partial \hat{r}^{2}}+\frac{3}{\hat{r}} \frac{\partial \Omega_{0}}{\partial \hat{r}}+2 \frac{\partial^{2} \Omega_{1}}{\partial \hat{r} \partial \tilde{r}}+\frac{3}{\hat{r}} \frac{\partial \Omega_{1}}{\partial \tilde{r}} .
$$

It can be shown that the solution $\Omega_{2}$ presents secular growth unless we set

$$
\frac{\partial A}{\partial \tau}=\frac{\partial^{2} A}{\partial \hat{r}^{2}}+\frac{3}{\hat{r}} \frac{\partial A}{\partial \hat{r}}, \quad \text { and } \quad \frac{\partial H}{\partial \tau}=\frac{\partial^{2} H}{\partial \hat{r}^{2}}+\frac{3}{\hat{r}} \frac{\partial H}{\partial \hat{r}} .
$$

The solutions are taken as

$$
A=\frac{D \exp \left(-\frac{\hat{r}^{2}}{B+4 \tau}\right)}{(B+4 \tau)^{2}}+C, \quad \text { and } \quad H=\frac{E \exp \left(-\frac{\hat{r}^{2}}{G+4 \tau}\right)}{(G+4 \tau)^{2}}+F
$$

where $B, C, D, E, F$ and $G$ are constants. We then impose that $\Omega_{0}$ at $t=\tau=0$ matches the profile (5.1). This implies $B=G=1, E=D=1 / 2, F=-C=-1 /(2 R o)$. Then, 
the solution of (5.7) can be found

$$
\Omega_{2}=-\frac{1}{\sqrt{\pi}}\left(\frac{\partial^{2} A}{\partial r^{2}}+\frac{3}{r} \frac{\partial A}{\partial r}+\frac{3}{4 r^{2}} A\right) \tilde{r} \sqrt{t} \exp \left(-\frac{\tilde{r}^{2}}{4 t}\right) .
$$

Finally, the complete solution for $\Omega$ up to order $R e^{-1}$, written back in terms of the original variables $r$ and $t$, reads

$$
\begin{aligned}
\Omega & =A \operatorname{erf}\left(\frac{\sqrt{R e}\left(r-r_{c}\right)}{2 \sqrt{t}}\right)+A-\frac{1}{R o}+\left[2 \frac{\partial A}{\partial r}+\frac{3}{r} A\right. \\
& \left.-\left(\frac{\partial^{2} A}{\partial r^{2}}+\frac{3}{r} \frac{\partial A}{\partial r}+\frac{3}{4 r^{2}} A\right)\left(r-r_{c}\right)\right] \sqrt{\frac{t}{\pi R e}} \exp \left(-\frac{R e\left(r-r_{c}\right)^{2}}{4 t}\right),
\end{aligned}
$$

where $A$ is given by 5.9 with the substitution $\hat{r}=r$ and $\tau=R e^{-1} t$. The azimuthal velocity profiles corresponding to (5.11) are plotted with dotted lines (viscous-KCO) in figure 6. The time in (5.11) has not been set to $t=60$ but to $t=20$. Indeed, since the profile (5.1) is the outcome of the centrifugal instability, we have assumed that it is virtually formed only at $t=40$ once the instability has almost ceased. These viscous profiles are in much better agreement with the DNS than the inviscid profile of Kloosterziel et al. (2007) and Carnevale et al. (2011). Besides, we emphasize that the profiles in the DNS and SL models are in excellent agreement.

\section{Conclusion}

We have studied the nonlinear growth of the centrifugal instability in an anticyclone with Gaussian angular velocity in rotating fluids for $R o=-4$. We have used an approach similar to the one behind the self-consistent model (Mantič-Lugo et al. 2014). Using Reynolds decompositions (Reynolds \& Hussain 1972) based upon a time average, MantičLugo et al. (2014) have separated the flow into mean flow and time harmonic fluctuations. These two components are coupled via Reynolds stresses in the mean flow equation and via the evolution of the mean flow in the fluctuation equation. In the present study on the centrifugal instability, we have used a spatial average instead of a time average and separated the flow into axially averaged mean flow and spatial harmonic fluctuation. Like for the self-consistent model, the fluctuation grows over an evolving mean flow while the mean flow is forced by the Reynolds stresses due to the fluctuations. Such semi-linear model with one harmonic is in very good agreement with DNS for $R e=800$ and $R e=2000$ concerning both the time evolution of the fluctuation amplitude and of the mean flow profiles. Including a second harmonic $2 k_{m}$ into the model improves slightly the predictions.

We have also compared the 'final' azimuthal velocity profile observed in the DNS and semi-linear models when the instability has disappeared to the inviscid profile proposed by Kloosterziel et al. (2007) based on homogenization of angular momentum towards a centrifugally stable flow. They agree except in the neighbourhood of the radius where the inviscid profile is discontinuous. To improve the prediction, we have computed asymptotically for large Reynolds number the viscous diffusion of the theoretical profile of Kloosterziel et al. (2007). The discontinuity is then smoothed and the predicted profiles are in much better agreement with the profile observed in the DNS and semi-linear models.

The main interest of the present semi-linear models is their simplicity which may enable a deeper understanding of the underlying physics. In addition, we emphasize that they are very cheap in terms of computational cost. Indeed, the computing time for a DNS with 
four processors takes 30 hours (elapsed real time) for 100 time units. In contrast, a run of the semi-linear model with one or two harmonics only takes $6 \mathrm{~min}$ or $10 \mathrm{~min}$, respectively with a single processor. This dramatic decrease on the computing time comes from the reduction of the problem to only few one-dimensional equations.

In the future, it would be interesting to investigate the connection between semi-linear models and amplitude equations derived from weakly nonlinear analyses.

Declaration of Interests. The authors report no conflict of interest.

\section{REFERENCES}

Batchelor, G. K. \& Gill, A. E. 1962 Analysis of the stability of axisymmetric jets. J. Fluid Mech. 14 (4), 529-551.

Billant, P. \& Gallaire, F. 2005 Generalized rayleigh criterion for non-axisymmetric centrifugal instabilities. J. Fluid Mech. 542, 365-379.

Carnevale, G. F., Kloosterziel, R. C., Orlandi, P. \& Van Sommeren, D. D. J. A. 2011 Predicting the aftermath of vortex breakup in rotating flow. J. Fluid Mech. 669, 90-119.

Deloncle, A., Billant, P. \& Chomaz, J.-M. 2008 Nonlinear evolution of the zigzag instability in stratified fluids: a shortcut on the route to dissipation. J. Fluid Mech. 599, 229-239.

Hecht, F. 2012 New development in freefem++. J. Numer. Math. 20 (3-4), 251-265.

Kloosterziel, R. C., Carnevale, G. F. \& Orlandi, P. 2007 Inertial instability in rotating and stratified fluids: barotropic vortices. J. Fluid Mech. 583, 379-412.

Kloosterziel, R. C. \& van Heijst, G. J. F. 1991 An experimental study of unstable barotropic vortices in a rotating fluid. J. Fluid Mech. 223, 1-24.

Lazar, A., Stegner, A. \& Heifetz, E. 2013 Inertial instability of intense stratified anticyclones. part 1. generalized stability criterion. J. Fluid Mech. 732, 457-484.

Mantič-Lugo, V., C., Arratia, C. \& Gallaire, F. 2014 Self-consistent mean flow description of the nonlinear saturation of the vortex shedding in the cylinder wake. Phys. Rev. Lett. 113, 084501.

Meliga, P. 2017 Harmonics generation and the mechanics of saturation in flow over an open cavity: a second-order self-consistent description. J. Fluid Mech. 826, 503-521.

Rayleigh, Lord 1917 On the dynamics of revolving fluids. Proc. R. Soc. A 93 (648), 148-154.

Reynolds, W. C. \& Hussain, A. K. M. F. 1972 The mechanics of an organized wave in turbulent shear flow. part 3. theoretical models and comparisons with experiments. $J$. Fluid Mech. 54 (2), 263-288.

Sмyth, W. D. \& McWilliams, J. C. 1998 Instability of an axisymmetric vortex in a stably stratified, rotating environment. Theor. Comp. Fluid Dyn. 11 (3-4), 305-322.

Synge, J. L. 1933 The stability of heterogeneous liquids. Trans. R. Soc. Canada 27, 1.

Yim, E., Billant, P. \& Ménesguen, C. 2016 Stability of an isolated pancake vortex in continuously stratified-rotating fluids. J. Fluid Mech. 801, 508-553. 